\title{
Construction of a measure of noncompactness in Sobolev spaces with an application to functional integral-differential equations
}

\author{
Mahnaz Khanehgir $^{1} \cdot$ Reza Allahyari ${ }^{1} \cdot$ Nayereh Gholamian $^{2}$
}

Received: 22 September 2016/Accepted: 28 December 2017/Published online: 12 January 2018

(C) The Author(s) 2018. This article is an open access publication

\begin{abstract}
In this paper, first we introduce a measure of noncompactness in the Sobolev space $W^{k, 1}(\Omega)$ and then, as an application, we study the existence of solutions for a class of the functional integral-differential equations using Darbo's fixed point theorem associated with this new measure of noncompactness. Further, two examples are presented to verify the effectiveness and applicability of our main results.
\end{abstract}

Keywords Darbo's fixed point theorem · Integral-differential equation · Measure of noncompactness · Sobolev space

Mathematical Subject Classification 45J05 $\cdot 47 \mathrm{H} 08 \cdot 47 \mathrm{H} 10$

\section{Introduction}

Sobolev spaces [11], i.e., the class of functions with derivatives in $L^{p}$, play an outstanding role in the modern analysis. In the last decades, there has been increasing attempts to study these spaces. Their importance comes from the fact that solutions of partial differential equations are naturally found in Sobolev spaces. They also highlighted in approximation theory, calculus of variation, differential geometry, spectral theory etc.

On the other hand, integral-differential equations (IDE) have a great deal of applications in some branches of sciences. It arises especially in a variety of models from applied mathematics, biological science, physics and another phenomenon, such as the theory of electrodynamics, electromagnetic, fluid dynamics, heat and oscillating magnetic, etc. $[9,12,18,21,24]$. There have appeared recently a number of

Reza Allahyari

rezaallahyari@mshdiau.ac.ir

Mahnaz Khanehgir

khanehgir@mshdiau.ac.ir

Nayereh Gholamian

nayereh1183@yahoo.com

1 Department of Mathematics, Mashhad Branch, Islamic Azad University, Mashhad, Iran

2 Department of Mathematics, Shirvan Branch, Islamic Azad University, Shirvan, Iran interesting papers $[2,6,10,19,22,23,27]$ on the solvability of various integral equations with help of measures of noncompactness.

The first such measure was defined by Kuratowski [25]. Next, Banaś et al. [8] proposed a generalization of this notion which is more convenient in the applications. The technique of measures of noncompactness is frequently applicable in several branches of nonlinear analysis, in particular the technique turns out to be a very useful tool in the existence theory for several types of integral and integral-differential equations. Furthermore, they are often used in the functional equations, fractional partial differential equations, ordinary and partial differential equations, operator theory and optimal control theory $[1,3,7,13$, 15-17, 26, 28, 29]. The most important application of measures of noncompactness in the fixed point theory is contained in the Darbo's fixed point theorem $[4,5]$.

Now, in this paper, we introduce a new measure of noncompactness in the Sobolev space $W^{k, 1}(\Omega)$ as a more effective approach. Then, we study the problem of existence of solutions of the functional integral-differential equation

$$
\begin{aligned}
u(x)= & p(x)+q(x) u(x) \\
& +\int_{\Omega} k(x, y) g\left(y, u(y), \frac{\partial u}{\partial x_{1}}(y), \ldots, \frac{\partial u}{\partial x_{n}}(y), T u(y)\right) \mathrm{d} y .
\end{aligned}
$$

We provide some notations, definitions and auxiliary facts which will be needed further on. 
Throughout this paper, $\mathbb{R}_{+}$indicates the interval $[0,+\infty)$ and for the Lebesgue measurable subset $D$ of $\mathbb{R}, m(D)$ denotes the Lebesgue measure of $D$. Moreover, let $L^{1}(D)$ be the space of all Lebesgue integrable functions $f$ on $D$ equipped with the standard norm $\|f\|_{L^{1}(D)}=\int_{D}|f(x)| \mathrm{d} x$.

Let $(E,\|\cdot\|)$ be a real Banach space with zero element 0 . The symbol $\bar{B}(x, r)$ stands for the closed ball centered at $x$ with radius $r$ and put $\bar{B}_{r}=\bar{B}(0, r)$. Denote by $\mathfrak{M}_{E}$ the family of nonempty and bounded subsets of $E$ and by $\mathfrak{N}_{E}$ its subfamily consisting of all relatively compact sets of $E$. For a nonempty subset $X$ of $E$, the symbols $\bar{X}$ and $\operatorname{Conv} X$ will denote the closure and the closed convex hull of $X$, respectively.

Definition 1.1 [8] A mapping $\mu: \mathfrak{M}_{\mathfrak{E}} \rightarrow \mathbb{R}_{+}$is said to be a measure of noncompactness in $E$ if it satisfies the following conditions:

$1^{\circ}$ The family $\operatorname{ker} \mu=\left\{X \in \mathfrak{M}_{\mathfrak{E}} \mu(\mathfrak{X})=\right\}$ is nonempty and $\operatorname{ker} \mu \subset \mathfrak{N}_{\mathfrak{E}}$.

$2^{\circ} X \subset Y \Rightarrow \mu(X) \leq \mu(Y)$.

$3^{\circ} \mu(\bar{X})=\mu(X)$.

$4^{\circ} \mu(\operatorname{Conv} X)=\mu(X)$.

$5^{\circ} \mu(\lambda X+(1-\lambda) Y) \leq \lambda \mu(X)+(1-\lambda) \mu(Y)$ for $\lambda \in$ $[0,1]$.

$6^{\circ}$ If $\left\{X_{n}\right\}$ is a sequence of closed sets from $\mathfrak{M}_{\mathfrak{E}}$ such that $X_{n+1} \subset X_{n}$ for $n=1,2, \ldots$ and if $\lim _{n \rightarrow \infty} \mu\left(X_{n}\right)=0$ then the set $X_{\infty}=\bigcap_{n=1}^{\infty} X_{n}$ is nonempty. A measure of noncompactness $\mu$ is said to be regular if it additionally satisfies the following conditions:

$7^{\circ} \mu(X \bigcup Y)=\max \{\mu(X), \mu(Y)\}$.

$8^{\circ} \mu(X+Y) \leq \mu(X)+\mu(Y)$.

$9^{\circ} \mu(\lambda X)=|\lambda| \mu(X)$ for $\lambda \in \mathbb{R}$.

$10^{\circ} \operatorname{ker} \mu=\mathfrak{N}_{\mathfrak{E}}$.

In what follows, we recall the well known Darbo's fixed point theorem.

Theorem 1.2 [13] Let $\Omega$ be a nonempty, bounded, closed and convex subset of a Banach space $E$ and let $F: \Omega \rightarrow \Omega$ be a continuous mapping such that there exists a constant $k \in[0,1)$ with the property

$\mu(F X) \leq k \mu(X)$,

for any nonempty subset $X$ of $\Omega$, where $\mu$ is a measure of noncompactness defined in E. Then, $F$ has a fixed point in the set $\Omega$.

\section{Construction of a measure of noncompactness in Sobolev spaces}

In this section, we introduce a measure of noncompactness in the Sobolev space $W^{k, 1}(\Omega)$.
Let $\Omega$ be a subset of $\mathbb{R}^{n}$ and $k \in \mathbb{N}$, we denote by $W^{k, 1}(\Omega)$ the space of functions $f$ which, together with all their distributional derivatives $D^{\alpha} f$ of order $|\alpha| \leq k$, belong to $L^{1}(\Omega)$. Here $\alpha=\left(\alpha_{1}, \ldots, \alpha_{n}\right)$ is a multi-index, i.e., each $\alpha_{j}$ is a nonnegative integer, $|\alpha|=\alpha_{1}+\cdots+\alpha_{n}$, and

$D^{\alpha}=\partial^{|\alpha|} / \partial x_{1}^{\alpha_{1}} \ldots \partial x_{n}^{\alpha_{n}}$.

Then, $W^{k, 1}(\Omega)$ is equipped with the complete norm $\|f\|_{k, 1}=\max _{0 \leq|\alpha| \leq k}\left\|D^{\alpha} f\right\|_{L^{1}(\Omega)}$.

We present the following theorem which characterizes the compact subsets of the Sobolev spaces.

Theorem 2.1 [20] A subset $\mathcal{F} \subset W^{k, 1}\left(\mathbb{R}^{n}\right)$ is totally bounded if, and only if, the following holds:

(i) $\mathcal{F}$ is bounded, i.e., there is some $M$ so that

$$
\int\left|D^{\alpha} f(x)\right| \mathrm{d} x<M, f \in \mathcal{F},|\alpha| \leq k
$$

(ii) For every $\varepsilon>0$ there is some $R$ so that

$$
\int_{\|x\|_{\mathbb{R}^{n}}>R}\left|D^{\alpha} f(x)\right| \mathrm{d} x<\varepsilon, f \in \mathcal{F},|\alpha| \leq k .
$$

(iii) For every $\varepsilon>0$ there is some $\rho>0$ so that

$$
\int_{\mathbb{R}^{n}}\left|D^{\alpha} f(x+y)-D^{\alpha} f(x)\right| \mathrm{d} x<\varepsilon, f \in \mathcal{F},|\alpha| \leq k,\|y\|_{\mathbb{R}^{n}}<\rho .
$$

Now, we are going to describe a measure of noncompactness in $W^{k, 1}(\Omega)$.

Theorem 2.2 Suppose $1 \leq k<\infty$ and $U$ is a bounded subset of $W^{k, 1}(\Omega)$. For $u \in U, \varepsilon>0$ and $0 \leq|\alpha| \leq k$, let

$$
\begin{aligned}
\omega^{T}(u, \varepsilon)= & \sup \left\{\left\|\mathcal{T}_{h} D^{\alpha} u-D^{\alpha} u\right\|_{L^{1}\left(B_{T}\right)}: h \in \Omega,\right. \\
& \left.\|h\|_{\mathbb{R}^{n}}<\varepsilon, 0 \leq|\alpha| \leq k\right\}, \\
\omega^{T}(U, \varepsilon)= & \sup \left\{\omega^{T}(u, \varepsilon): u \in U\right\}, \\
\omega^{T}(U)= & \lim _{\varepsilon \rightarrow 0} \omega^{T}(U, \varepsilon), \\
\omega(U)= & \lim _{T \rightarrow \infty} \omega^{T}(U), \\
d(U)= & \lim _{T \rightarrow \infty} \sup \left\{\left\|D^{\alpha} u\right\|_{L^{1}\left(\Omega \backslash B_{T}\right)}: u \in U, 0 \leq|\alpha| \leq k\right\},
\end{aligned}
$$

where $B_{T}=\left\{a \in \Omega:\|a\|_{\mathbb{R}^{n}} \leq T\right\}$ and $\mathcal{T}_{h} u(t)=u(t+h)$.

Then $\omega_{0}: \mathfrak{M}_{\left.\mathfrak{W}^{\mathfrak{f}}, \Omega\right)} \rightarrow \mathbb{R}$ given by

$\omega_{0}(U)=\omega(U)+d(U)$

defines a measure of noncompactness in $W^{k, 1}(\Omega)$.

Proof Take $U \in \mathfrak{M}_{\mathfrak{W}^{\mathrm{f}},(\Omega)}$ such that $\omega_{0}(U)=0$. Fix arbitrary $\alpha$ such that $0 \leq|\alpha| \leq k$. Let $\eta>0$ be arbitrary, since $\omega_{0}(U)=0$, 
$\lim _{T \rightarrow \infty} \lim _{\varepsilon \rightarrow 0} \omega^{T}(U, \varepsilon)=0$.

Thus, there exists small enough $\delta>0$ and large enough $T>0$ such that $\omega^{T}(U, \delta)<\eta$. This implies that

$\left\|\mathcal{T}_{h} D^{\alpha} u-D^{\alpha} u\right\|_{L^{1}\left(B_{T}\right)}<\eta$

for all $u \in U$ and $h \in \Omega$ such that $\|h\|_{\mathbb{R}^{n}}<\delta$. Since $\eta>0$ was arbitrary, we obtain

$\lim _{h \rightarrow 0}\left\|\mathcal{T}_{h} D^{\alpha} u-D^{\alpha} u\right\|_{L^{1}(\Omega)}=\lim _{h \rightarrow 0}$

$\lim _{T \rightarrow \infty}\left\|\mathcal{T}_{h} D^{\alpha} u-D^{\alpha} u\right\|_{L^{1}\left(B_{T}\right)}=0$.

Using again the fact that $\omega_{0}(U)=0$ we have

$\lim _{T \rightarrow \infty} \sup \left\{\left\|D^{\alpha} u\right\|_{L^{1}\left(\Omega \backslash B_{T}\right)}: u \in U\right\}=0$,

and so for $\varepsilon>0$ there exists large enough $T>0$ such that $\left\|D^{\alpha} u\right\|_{L^{1}\left(\Omega \backslash B_{T}\right)}<\varepsilon$ for all $u \in U$.

It follows then from Theorem 2.1 that $U$ is totally bounded. Thus, $1^{\circ}$ holds.

$2^{\circ}$ is obvious by the definition of $\omega_{0}$.

Now, we check that condition $3^{\circ}$ holds. For this purpose, suppose that $U \in \mathfrak{M}_{\mathfrak{W}^{\mathrm{t}},(\Omega)}$ and $\left\{u_{n}\right\} \subset U$ such that $u_{n} \rightarrow$ $u \in \bar{U}$ in $W^{k, 1}(\Omega)$. From the definition of $\omega^{T}(U, \varepsilon)$, we have

$$
\left\|\mathcal{T}_{h} D^{\alpha} u_{n}-D^{\alpha} u_{n}\right\|_{L^{1}\left(B_{T}\right)} \leq \omega^{T}(U, \varepsilon),
$$

for any $n \in \mathbb{N}, T>0$ and $h \in \Omega$ with $\|h\|_{\mathbb{R}^{n}}<\varepsilon$. Letting $n \rightarrow \infty$, we get

$\left\|\mathcal{T}_{h} D^{\alpha} u-D^{\alpha} u\right\|_{L^{1}\left(B_{T}\right)} \leq \omega^{T}(U, \varepsilon)$,

for any $T>0$ and $h \in \Omega$ with $\|h\|_{\mathbb{R}^{n}}<\varepsilon$. Hence

$\lim _{T \rightarrow \infty \varepsilon \rightarrow 0} \lim _{\varepsilon} \omega^{T}(\bar{U}, \varepsilon) \leq \lim _{T \rightarrow \infty \varepsilon \rightarrow 0} \lim _{\varepsilon} \omega^{T}(U, \varepsilon)$.

This concludes that $\omega(\bar{U}) \leq \omega(U)$. Similarly, we can show that

$d(\bar{U}) \leq d(U)$,

and thus

$\omega_{0}(\bar{U}) \leq \omega_{0}(U)$.

From (3) and $2^{\circ}$ we obtain $\omega_{0}(\bar{U})=\omega_{0}(U)$.

$4^{\circ}$ follows directly from $D^{\alpha}[\operatorname{Conv}(U)]=\operatorname{Conv}\left(D^{\alpha} U\right)$ and hence is omitted.

The proof of condition $5^{\circ}$ can be obtained by using the equality

$D^{\alpha}\left(\lambda u_{1}+(1-\lambda) u_{2}\right)=\lambda D^{\alpha} u_{1}+(1-\lambda) D^{\alpha} u_{2}$,

for all $\lambda \in[0,1], u_{1} \in X$ and $u_{2} \in Y$.
It remains only to verify $6^{\circ}$, suppose that $\left\{U_{n}\right\}$ is a sequence of closed and nonempty sets of $\mathfrak{M}_{\mathfrak{W}^{\mathrm{t}},(\Omega)}$ such that $U_{n+1} \subset U_{n}$ for $n=1,2, \ldots$, and $\lim _{n \rightarrow \infty} \omega_{0}\left(U_{n}\right)=0$. Now, for any $n \in \mathbb{N}$, take $u_{n} \in U_{n}$ and set $\mathcal{G}=\overline{\left\{u_{n}\right\}}$.

Claim: $\mathcal{G}$ is a compact set in $W^{k, 1}(\Omega)$.

Let $\varepsilon>0$ be fixed, since $\lim _{n \rightarrow \infty} \omega_{0}\left(U_{n}\right)=0$, there exists sufficiently large $m_{1} \in \mathbb{N}$ such that $\omega_{0}\left(U_{m_{1}}\right)<\varepsilon$. Hence, we can find small enough $\delta_{1}>0$ and large enough $T_{1}>0$ such that $\omega^{T_{1}}\left(U_{m_{1}}, \delta_{1}\right)<\varepsilon$ and $d\left(U_{m_{1}}\right)<\varepsilon$. Therefore,

$\left\|\mathcal{T}_{h} D^{\alpha} u_{n}-D^{\alpha} u_{n}\right\|_{L^{1}\left(B_{T_{1}}\right)}<\varepsilon$,

and

$\left\|D^{\alpha} u_{n}\right\|_{L^{1}\left(\Omega \backslash B_{T_{1}}\right)}<\varepsilon$,

for all $n>m_{1}, 0 \leq|\alpha| \leq k$ and $h \in \Omega$ with $\|h\|_{\mathbb{R}^{n}}<\delta_{1}$. Thus we have

$$
\begin{aligned}
& \left\|\mathcal{T}_{h} D^{\alpha} u_{n}-D^{\alpha} u_{n}\right\|_{L^{1}(\Omega)} \\
\leq & \left\|\mathcal{T}_{h} D^{\alpha} u_{n}-D^{\alpha} u_{n}\right\|_{L^{1}\left(B_{T_{1}}\right)}+\left\|\mathcal{T}_{h} D^{\alpha} u_{n}-D^{\alpha} u_{n}\right\|_{L^{1}\left(\Omega \backslash B_{T_{1}}\right)} \\
\leq & \left\|\mathcal{T}_{h} D^{\alpha} u_{n}-D^{\alpha} u_{n}\right\|_{L^{1}\left(B_{T_{1}}\right)}+\left\|\mathcal{T}_{h} D^{\alpha} u_{n}\right\|_{L^{1}\left(\Omega \backslash B_{T_{1}}\right)} \\
& +\left\|D^{\alpha} u_{n}\right\|_{L^{1}\left(\Omega \backslash B_{T_{1}}\right)} \\
< & 3 \varepsilon
\end{aligned}
$$

On the other hand, we know that the set $\left\{u_{1}, u_{2}, \ldots, u_{m_{1}}\right\}$ is compact, hence there exist $\delta_{2}>0$ and $T_{2}>0$ such that

$\left\|\mathcal{T}_{h} D^{\alpha} u_{n}-D^{\alpha} u_{n}\right\|_{L^{1}\left(B_{T_{2}}\right)}<\varepsilon$

for all $n=1,2, \ldots, m_{1}, \quad 0 \leq|\alpha| \leq k$ and $h \in \Omega$ with $\|h\|_{\mathbb{R}^{n}}<\delta_{2}$.

Furthermore,

$\left\|D^{\alpha} u_{n}\right\|_{L^{1}\left(\Omega \backslash B_{T_{2}}\right)}<\varepsilon$,

which implies that

$\left\|\mathcal{T}_{h} D^{\alpha} u_{n}-D^{\alpha} u_{n}\right\|_{L^{1}(\Omega)}<3 \varepsilon$,

for all $n=1,2, \ldots, m_{1}$.

Thus,

$\left\|\mathcal{T}_{h} D^{\alpha} u_{n}-D^{\alpha} u_{n}\right\|_{L^{1}(\Omega)}<3 \varepsilon$,

and

$\left\|D^{\alpha} u_{n}\right\|_{L^{1}\left(\Omega \backslash B_{T}\right)}<\varepsilon<3 \varepsilon$,

for all $n \in \mathbb{N},\|h\|_{\mathbb{R}^{n}}<\min \left\{\delta_{1}, \delta_{2}\right\}$ and $T=\max \left\{T_{1}, T_{2}\right\}$. Therefore, all the hypotheses of Theorem 2.1 are satisfied, that completes the proof of the claim.

Using the above claim, there exists a subsequence $\left\{u_{n_{j}}\right\}$ and $u_{0} \in W^{k, 1}(\Omega)$ such that $u_{n_{j}} \rightarrow u_{0}$. Since $u_{n} \in U_{n}$, $U_{n+1} \subset U_{n}$ and $U_{n}$ is closed for all $n \in \mathbb{N}$, we yield 
$u_{0} \in \bigcap_{n=1}^{\infty} U_{n}=U_{\infty}$

that finishes the proof of $6^{\circ}$.

We now investigate the regularity of $\omega_{0}$.

Theorem 2.3 The measure of noncompactness $\omega_{0}$ defined in (2) is regular.

Proof Suppose that $X, Y \in \mathfrak{M}_{\mathfrak{W}^{\ddagger},(\Omega)}$. First, notice that for all $\varepsilon>0, \lambda \in \mathbb{R}$ and $T>0$ we have

$$
\begin{aligned}
\omega^{T}(X \cup Y, \varepsilon) & =\max \left\{\omega^{T}(X, \varepsilon), \omega^{T}(Y, \varepsilon)\right\}, \\
\omega^{T}(X+Y, \varepsilon) \leq & \omega^{T}(X, \varepsilon)+\omega^{T}(Y, \varepsilon), \\
\omega^{T}(\lambda X, \varepsilon)= & |\lambda| \omega^{T}(X, \varepsilon), \\
\sup _{u \in X \cup Y}\left\|D^{\alpha} u\right\|_{L^{1}\left(\Omega \backslash B_{T}\right)} & =\max \left\{\sup _{u \in X}\left\|D^{\alpha} u\right\|_{L^{1}\left(\Omega \backslash B_{T}\right)},\right. \\
& \left.\sup _{u \in Y}\left\|D^{\alpha} u\right\|_{L^{1}\left(\Omega \backslash B_{T}\right)}\right\}, \\
\sup _{u \in X+Y}\left\|D^{\alpha} u\right\|_{L^{1}\left(\Omega \backslash B_{T}\right)} \leq & \sup _{u \in X}\left\|D^{\alpha} u\right\|_{L^{1}\left(\Omega \backslash B_{T}\right)} \\
& +\sup _{u \in Y}\left\|D^{\alpha} u\right\|_{L^{1}\left(\Omega \backslash B_{T}\right)}, \\
\sup _{u \in \lambda X}\left\|D^{\alpha} u\right\|_{L^{1}\left(\Omega \backslash B_{T}\right)}= & |\lambda| \sup _{u \in X}\left\|D^{\alpha} u\right\|_{L^{1}\left(\Omega \backslash B_{T}\right)} .
\end{aligned}
$$

Then, the hypotheses $7^{\circ}-9^{\circ}$ hold. Next, we show that $10^{\circ}$ holds. Take $U \in \mathfrak{N}_{\mathfrak{W}^{\ddagger},(\Omega)}$. Thus, the closure of $U$ in $W^{k, 1}(\Omega)$ is compact. By Theorem 2.1, for all $|\alpha| \leq k$ and for all $\varepsilon>0$, there exists $T>0$ such that $\left\|D^{\alpha} u\right\|_{L^{1}\left(\Omega \backslash B_{T}\right)}<\varepsilon$ for all $u \in U$, and there exists $\delta>0$ such that $\| \mathcal{T}_{h} D^{\alpha} u-$ $D^{\alpha} u \|_{L^{1}\left(B_{T}\right)}<\varepsilon$ for all $h \in \Omega$ with $\|h\|_{\mathbb{R}^{n}}<\delta$. Then, for all $u \in U$ we have

$$
\begin{gathered}
\omega^{T}(u, \delta)=\sup \left\{\left\|\mathcal{T}_{h} D^{\alpha} u-D^{\alpha} u\right\|_{L^{1}\left(B_{T}\right)}: h \in \Omega,\right. \\
\left.\|h\|_{\mathbb{R}^{n}}<\delta\right\} \leq \varepsilon .
\end{gathered}
$$

Therefore,

$\omega^{T}(U, \delta)=\sup \left\{\left\|\omega^{T}(u, \delta)\right\|: u \in U\right\}=0$.

It yields that

$\omega(U)=\lim _{T \rightarrow \infty} \lim _{\delta \rightarrow 0} \omega^{T}(U, \delta)=0$.

Furthermore,

$d(U)=\lim _{T \rightarrow \infty} \sup \left\{\left\|D^{\alpha} u\right\|_{L^{1}\left(\Omega \backslash B_{T}\right)}: u \in U\right\}=0$.

Then $\omega_{0}(U)=0$ and $\operatorname{ker}\left(\omega_{0}\right)=\mathfrak{P}_{\mathfrak{W}^{\mathrm{f}},(\Omega)}$.

Theorem 2.4 Let $Q=\left\{u \in W^{k, 1}\left(\mathbb{R}^{n}\right):\|u\|_{1,1} \leq 1\right\}$. Then $\omega_{0}(Q)=3$.

Proof Applying the same strategy as ([4], Theorem [14]), we observe that $\omega_{0}(Q) \leq 3$. It remains to verify $\omega_{0}(Q) \geq 3$. For any $k \in \mathbb{N}$, there exists $E_{k} \subset \mathbb{R}^{n}$ such that $m\left(E_{k}\right)=\frac{1}{10 k}$,
$\operatorname{diam}\left(E_{k}\right) \leq \frac{1}{k}, E_{k} \cap B_{k}=\emptyset$ and $E_{k} \subset B_{2 k}$. Define $f_{k}: \mathbb{R}^{n} \rightarrow$ $\mathbb{R}$ by

$f_{k}(x)=\left\{\begin{array}{cc}10 k, & x \in E_{k}, \\ 0, & \text { otherwise. }\end{array}\right.$

In addition, observe that $\left\|f_{k}\right\|_{1,1}=1, \| D^{\alpha} \mathcal{T}_{\beta_{k}} f_{k}-$ $D^{\alpha} f_{k} \|_{L^{1}\left(B_{2 k}\right)}=2$ and

$\left\|D^{\alpha} f_{k}\right\|_{L^{1}\left(\mathbb{R}^{n} \backslash B_{k}\right)}=1 \quad$ for all $k \in \mathbb{N}$, where $\beta_{k}=$ $\left(\frac{1}{k}, \ldots, \frac{1}{k}\right) \in \mathbb{R}^{n}$. Thus, we conclude that $\omega_{0}(Q) \geq \omega$ $0\left(\left\{f_{k}\right\}\right)=3$.

\section{Application}

In this section, we study the existence of solutions for some functional integral-differential equations. We also provide some illustrative examples to verify effectiveness and applicability of our results.

We start with some preliminaries which we need in subsequence.

Lemma 3.1 [14] Let $\Omega$ be a Lebesgue measurable subset of $\mathbb{R}^{n}$ and $1 \leq p \leq \infty$. If $\left\{f_{n}\right\}$ is convergent to $f$ in the $L^{p}$ norm, then there is a subsequence $\left\{f_{n_{k}}\right\}$ which converges to $f$ a.e., and there is $g \in L^{p}(\Omega), g \geq 0$, such that

$\left|f_{n_{k}}(x)\right| \leq g(x)$ for a.e. $\mathrm{x} \in \Omega$.

Definition 3.2 [4] We say that a function $f: \mathbb{R}^{n} \times \mathbb{R}^{m} \rightarrow$ $\mathbb{R}$ satisfies the Carathéodory conditions if the function $f(., u)$ is measurable for any $u \in \mathbb{R}^{m}$ and the function $f(x,$. is continuous for almost all $x \in \mathbb{R}^{n}$.

Let $\Omega$ be a subset of $\mathbb{R}^{n}$ and $k \in \mathbb{N}$, we denote by $B C^{k}(\Omega)$ the space of functions $f$ which are bounded and $k$ times continuously differentiable on $\Omega$ with the standard norm

$\|f\|_{B C^{k}(\Omega)}=\max _{0 \leq|\alpha| \leq k}\left\|D^{\alpha} f\right\|_{u}$,

where $\left\|D^{\alpha} f\right\|_{u}=\sup \left\{\left|D^{\alpha} f(x)\right|: x \in \Omega\right\}$.

Theorem 3.3 Let $\Omega$ be a subset of $\mathbb{R}^{n}$ with $m(\Omega)<\infty$. Assume that the following conditions are satisfied:

(i) $p \in W^{1,1}(\Omega), q \in B C^{1}(\Omega)$ and

$$
\lambda:=\sup \left\{\|q\|_{u}+\left\|\frac{\partial q}{\partial x_{i}}\right\|_{u}: i=1, \ldots, n\right\}<1 \text {. }
$$

(ii) $g: \Omega \times \mathbb{R}^{n+2} \rightarrow \mathbb{R}$ satisfies the Carath éodory conditions and there exist a bounded continuous function $a: \Omega \rightarrow \mathbb{R}_{+}$with $|a(x)| \leq M$ for all $x \in \Omega$ and some $M>0$ and a concave, lower semi- 
continuous and nondecreasing function $\zeta: \mathbb{R}_{+} \rightarrow$ $\mathbb{R}_{+}$such that

$$
\left|g\left(x, u_{0}, u_{1}, \ldots, u_{n+1}\right)\right| \leq a(x) \zeta\left(\max _{0 \leq i \leq n+1}\left|u_{i}\right|\right) .
$$

(iii) $\quad k: \Omega \times \Omega \rightarrow \mathbb{R}$ satisfies the Carath éodory conditions and has a derivative of order 1 with respect to the first argument. Moreover, there exist $g_{1}, g_{3} \in W^{1,1}(\Omega)$ and $g_{2} \in L^{\infty}(\Omega)$ such that

$$
\begin{aligned}
|k(x, y)| & \leq g_{1}(x) g_{2}(y),\left|k\left(x_{1}, y\right)-k\left(x_{2}, y\right)\right| \\
& \leq g_{2}(y)\left|g_{3}\left(x_{1}\right)-g_{3}\left(x_{2}\right)\right|,
\end{aligned}
$$

and

$\left|\frac{\partial k}{\partial x_{i}}(x, y)\right| \leq g_{1}(x) g_{2}(y),\left|\frac{\partial k}{\partial x_{i}}\left(x_{1}, y\right)-\frac{\partial k}{\partial x_{i}}\left(x_{2}, y\right)\right|$

$$
\leq g_{2}(y)\left|g_{3}\left(x_{1}\right)-g_{3}\left(x_{2}\right)\right| \text {, }
$$

for almost $x, y, x_{1}, x_{2} \in \Omega$ and $1 \leq i \leq n$.

(iv) There exists a positive solution $r_{0}$ of the inequality

$$
\begin{aligned}
& \|p\|_{1,1}+\lambda r+\operatorname{Mm}(\Omega)\left\|g_{1}\right\|_{L^{1}(\Omega)}\left\|g_{2}\right\|_{L^{\infty}} \\
& \zeta\left(\frac{1}{m(\Omega)}\|u\|_{1,1}\right) \leq r .
\end{aligned}
$$

(v) $\quad T: W^{1,1}(\Omega) \rightarrow L^{1}(\Omega)$ is a continuous operator such that for any $x \in W^{1,1}(\Omega)$ we have

$$
\|T(x)\|_{L^{1}(\Omega)} \leq\|x\|_{1,1} \text {. }
$$

Then, the functional integral-differential equation

$$
\begin{aligned}
u(x)= & p(x)+q(x) u(x)+\int_{\Omega} k(x, y) g\left(y, u(y), \frac{\partial u}{\partial x_{1}}(y), \ldots,\right. \\
& \left.\frac{\partial u}{\partial x_{n}}(y), T u(y)\right) \mathrm{d} y
\end{aligned}
$$

has at least one solution in the space $W^{1,1}(\Omega)$.

Proof We define the operator $F: W^{1,1}(\Omega) \rightarrow W^{1,1}(\Omega)$ by

$$
\begin{aligned}
F u(x)= & p(x)+q(x) u(x)+\int_{\Omega} k(x, y) g\left(y, u(y), \frac{\partial u}{\partial x_{1}}(y),\right. \\
& \left.\ldots, \frac{\partial u}{\partial x_{n}}(y), T u(y)\right) \mathrm{d} y .
\end{aligned}
$$

Obviously, $F u$ is measurable for any $u \in W^{1,1}(\Omega)$. Also, for any $x \in \Omega$ we have

$$
\begin{aligned}
\frac{\partial(F u)}{\partial x_{i}}(x)= & \frac{\partial p}{\partial x_{i}}(x)+\frac{\partial q}{\partial x_{i}}(x) u(x)+q(x) \frac{\partial u}{\partial x_{i}}(x) \\
& +\int_{\Omega} \frac{\partial k}{\partial x_{i}}(x, y) g\left(y, u(y), \frac{\partial u}{\partial x_{1}}(y), \ldots,\right. \\
& \left.\frac{\partial u}{\partial x_{n}}(y), T u(y)\right) \mathrm{d} y
\end{aligned}
$$

and $F u$ has measurable derivatives. We show that, $F u \in W^{1,1}(\Omega)$. Using our assumptions, for arbitrarily fixed $x \in \Omega$, we have

$$
\begin{aligned}
& |F u(x)| \leq|p(x)|+|q(x)||u(x)| \\
& +\mid \int_{\Omega} k(x, y) g\left(y, u(y), \frac{\partial u}{\partial x_{1}}(y), \ldots, \frac{\partial u}{\partial x_{n}}(y),\right. \\
& \quad(y)) \mathrm{d} y \mid .
\end{aligned}
$$

According to the Jensen's inequality, we deduce

$$
\begin{aligned}
& \|F u\|_{L^{1}(\Omega)} \leq\|p\|_{L^{1}(\Omega)}+\|q\|_{u}\|u\|_{L^{1}(\Omega)}+\operatorname{Mm}(\Omega) \| g_{1} \\
& \left\|_{L^{1}(\Omega)}\right\| g_{2} \|_{L^{\infty}} \zeta\left(\frac{1}{m(\Omega)}\|u\|_{1,1}\right) .
\end{aligned}
$$

By the same argument as above,

$$
\begin{gathered}
\left|\frac{\partial(F u)}{\partial x_{i}}(x)\right| \leq\left|\frac{\partial p}{\partial x_{i}}(x)\right|+\left|\frac{\partial q}{\partial x_{i}}(x)\right||u(x)|+|q(x)|\left|\frac{\partial u}{\partial x_{i}}(x)\right| \\
+\mid \int_{\Omega} \frac{\partial k}{\partial x_{i}}(x, y) g\left(y, u(y), \frac{\partial u}{\partial x_{1}}(y), \ldots,\right. \\
\left.\frac{\partial u}{\partial x_{n}}(y), T u(y)\right) \mathrm{d} y \mid
\end{gathered}
$$

and

$$
\begin{aligned}
\left\|\frac{\partial(F u)}{\partial x_{i}}\right\|_{L^{1}(\Omega)} \leq & \left\|\frac{\partial p}{\partial x_{i}}(x)\right\|_{L^{1}(\Omega)}+\left\|\frac{\partial q}{\partial x_{i}}\right\|_{u}\|u\|_{L^{1}(\Omega)}+\|q\|_{u} \\
& \left\|\frac{\partial u}{\partial x_{i}}\right\|_{L^{1}(\Omega)}+\operatorname{Mm}(\Omega)\left\|g_{1}\right\|_{L^{1}(\Omega)}\left\|g_{2}\right\|_{L^{\infty}} \\
& \zeta\left(\frac{1}{m(\Omega)}\|u\|_{1,1}\right) .
\end{aligned}
$$

Thus, we obtain

$\|F u\|_{1,1} \leq\|p\|_{1,1}+\lambda\|u\|_{1,1}+\operatorname{Mm}(\Omega)\left\|g_{1}\right\|_{L^{1}(\Omega)}\left\|g_{2}\right\|_{L^{\infty}}$

$\zeta\left(\frac{1}{m(\Omega)}\|u\|_{1,1}\right)$.

Due to (11) and using condition (iv), we derive that $F$ is a mapping from $\bar{B}_{r_{0}}$ into $\bar{B}_{r_{0}}$. Now, we show that the map $F$ is continuous. Let $\left\{u_{m}\right\}$ be an arbitrary sequence in $W^{1,1}(\Omega)$ which converges to $u \in W^{1,1}(\Omega)$. By Lemma 3.1 there is a subsequence $\left\{u_{m_{k}}\right\}$ which converges to $u$ a.e., $\left\{\frac{\partial u_{m_{k}}}{\partial x_{i}}\right\}$ converges to $\left\{\frac{\partial u}{\partial x_{i}}\right\}$ a.e., $\left\{T u_{m_{k}}\right\}$ converges to $T u$ a.e. and there is $h \in L^{1}(\Omega), h \geq 0$, such that

$$
\begin{aligned}
& \max \left\{\left|u_{m_{k}}(y)\right|,\left|\frac{\partial u_{m_{k}}}{\partial x_{1}}(y)\right|,\left|\frac{\partial u_{m_{k}}}{\partial x_{2}}(y)\right|, \ldots,\left|T u_{m_{k}}(y)\right|\right\} \leq h(y) \\
& \text { for } \quad \text { a.e. } \quad \mathrm{y} \in \Omega .
\end{aligned}
$$

Since $u_{m_{k}} \rightarrow u$ almost everywhere and $g$ satisfies the Carathéodory conditions, it follows that 
$g\left(y, u_{m_{k}}(y), \frac{\partial u_{m_{k}}}{\partial x_{1}}(y), \ldots, T u_{m_{k}}(y)\right) \rightarrow g\left(y, u(y), \frac{\partial u}{\partial x_{1}}(y), \ldots, T u(y)\right)$,

for almost all $y \in \Omega$.

From condition (ii) we have

$g\left(y, u_{m_{k}}(y), \frac{\partial u_{m_{k}}}{\partial x_{1}}(y), \ldots, T u_{m_{k}}(y)\right) \leq a(y) \zeta(h(y))$ for $\quad$ a.e. $\quad \mathrm{y} \in \Omega$.

As a consequence of the Lebesgue's Dominated Convergence Theorem, it yields that

$\int g\left(y, u_{m_{k}}(y), \frac{\partial u_{m_{k}}}{\partial x_{1}}(y), \ldots, T u_{m_{k}}(y)\right) \mathrm{d} y \rightarrow \int g(y, u(y)$,

$\left.\frac{\partial u}{\partial x_{1}}(y), \ldots, T u(y)\right) \mathrm{d} y$,

for almost all $y \in \Omega$. Inequality (12) and condition (iii) imply that

$\left\|F u_{m_{k}}-F u\right\|_{1,1} \rightarrow 0$ and $\left\|\frac{\partial \mathrm{Fu}_{\mathrm{m}_{\mathrm{k}}}}{\partial \mathrm{x}_{\mathrm{i}}}-\frac{\partial \mathrm{Fu}}{\partial \mathrm{x}_{\mathrm{i}}}\right\|_{1,1} \rightarrow 0$ as

$k \rightarrow \infty \quad(1 \leq i \leq n)$.

Therefore, $F: W^{1,1}(\Omega) \longrightarrow W^{1,1}(\Omega)$ is continuous.

To finish, the proof we have to verify that condition (1) is satisfied. We fix arbitrary $T>0$ and $\varepsilon>0$. Let $U$ be a nonempty and bounded subset of $\bar{B}_{r_{0}}$. Choose $u \in U$ and $x, h \in B_{T}$ with $\|h\|_{\mathbb{R}^{n}} \leq \varepsilon$, then we have

$$
\begin{aligned}
\int_{B_{T}} \mid & F u(x)-F u(x+h) \mid \mathrm{d} x \\
\leq & \int_{B_{T}}|p(x)-p(x+h)| \mathrm{d} x \\
+ & \int_{B_{T}}|q(x)-q(x+h)||u(x)| \mathrm{d} x \\
& +\int_{B_{T}}|q(x+h)||u(x)-u(x+h)| \mathrm{d} x \\
& +\int_{B_{T}} \int_{\Omega}|k(x, y)-k(x+h, y)| \mid g(y, u(y), \\
& \left.\frac{\partial u}{\partial x_{1}}(y), \ldots, \frac{\partial u}{\partial x_{n}}(y), T u(y)\right) \mid \mathrm{d} y \mathrm{~d} x \\
\leq & \omega^{T}(p, \varepsilon)+\int_{B_{T}}|q(x)-q(x+h) \| u(x)| \mathrm{d} x+\lambda \omega^{T}(U, \varepsilon) \\
& +M m(\Omega)\left\|g_{2}\right\|_{L^{\infty}} \zeta\left(\frac{1}{m(\Omega)}\|u\|_{1,1}\right) \omega^{T}\left(g_{3}, \varepsilon\right) .
\end{aligned}
$$

Obviously, $\omega^{T}(p, \varepsilon) \rightarrow 0, \omega^{T}\left(g_{3}, \varepsilon\right) \rightarrow 0$ and by continuity of $q$,

$$
\int_{B_{T}}|q(x)-q(x+h)||u(x)| \mathrm{d} x \rightarrow 0,
$$

as $\varepsilon \rightarrow 0$. Then the right hand side of (13) tends to $\lambda \omega^{T}(U)$ as $\varepsilon \rightarrow 0$.
By a similar argument and using condition (i), for each $i=1, \ldots, n$, we get

$$
\begin{aligned}
& \int_{B_{T}}\left|\frac{\partial(F u)}{\partial x_{i}}(x)-\frac{\partial(F u)}{\partial x_{i}}(x+h)\right| \mathrm{d} x \\
& \leq \int_{B_{T}}\left|\frac{\partial p}{\partial x_{i}}(x)-\frac{\partial p}{\partial x_{i}}(x+h)\right| \mathrm{d} x+\int_{B_{T}} \mid \frac{\partial q}{\partial x_{i}}(x) \\
& -\frac{\partial q}{\partial x_{i}}(x+h)|| u(x) \mid \mathrm{d} x \\
& +\int_{B_{T}}\left|\frac{\partial q}{\partial x_{i}}(x+h) \| u(x)-u(x+h)\right| \mathrm{d} x \\
& +\int_{B_{T}}\left|q(x) \| \frac{\partial u}{\partial x_{i}}(x)-\frac{\partial u}{\partial x_{i}}(x+h)\right| \mathrm{d} x \\
& +\int_{B_{T}}\left|\frac{\partial u}{\partial x_{i}}(x+h) \| q(x)-q(x+h)\right| \mathrm{d} x \\
& +\int_{B_{T}} \int_{\Omega}\left|\frac{\partial k}{\partial x_{i}}(x, y)-\frac{\partial k}{\partial x_{i}}(x+h, y)\right| \\
& \times\left|g\left(y, u(y), \frac{\partial u}{\partial x}(y), \ldots, \frac{\partial u}{\partial x_{n}}(y), T u(y)\right)\right| \mathrm{d} y \mathrm{~d} x \\
& \leq \omega^{T}(p, \varepsilon)+\int_{B_{T}}\left|\frac{\partial q}{\partial x_{i}}(x)-\frac{\partial q}{\partial x_{i}}(x+h) \| u(x)\right| \mathrm{d} x \\
& +\lambda \omega^{T}(U, \varepsilon) \\
& +\int_{B_{T}}\left|\frac{\partial u}{\partial x_{i}}(x+h) \| q(x)-q(x+h)\right| \mathrm{d} x \\
& +\operatorname{Mm}(\Omega)\left\|g_{2}\right\|_{L^{\infty}} \zeta\left(\frac{1}{m(\Omega)}\|u\|_{1,1}\right) \omega^{T}\left(g_{3}, \varepsilon\right) \text {. }
\end{aligned}
$$

Applying the same reasoning as above, the right hand side of (14) tends to $\lambda \omega^{T}(U)$ as $\varepsilon \rightarrow 0$, too. Regarding to (13) and (14), and since $u$ is an arbitrary element of $U$, then $\omega^{T}(F U) \leq \lambda \omega^{T}(U)$. Letting $T \rightarrow \infty$, we deduce

$$
\omega(F U) \leq \lambda \omega(U) \text {. }
$$

Next, let us fix an arbitrary number $T>0$. Then, taking into account our hypotheses, for an arbitrary function $u \in$ $U$ we derive

$$
\begin{aligned}
\|F u\|_{L^{1}\left(\Omega \backslash B_{T}\right)} \leq & \|p\|_{L^{1}\left(\Omega \backslash B_{T}\right)}+\|q\|_{u}\|u\|_{L^{1}\left(\Omega \backslash B_{T}\right)} \\
& +M m(\Omega)\left\|g_{1}\right\|_{L^{1}\left(\Omega \backslash B_{T}\right)}\left\|g_{2}\right\|_{\left.L^{\infty}\left(\Omega \backslash B_{T}\right)\right)} \\
& \zeta\left(\frac{1}{m(\Omega)}\|u\|_{1,1}\right) .
\end{aligned}
$$

Now, since

$$
\|p\|_{L^{1}\left(\Omega \backslash B_{T}\right)} \rightarrow 0, \quad\left\|g_{1}\right\|_{L^{1}\left(\Omega \backslash B_{T}\right)} \rightarrow 0 \quad \text { as } \quad T \rightarrow \infty,
$$

then

$\lim _{T \rightarrow \infty}\|F u\|_{L^{1}\left(\Omega \backslash B_{T}\right)} \leq \lambda d(U)$.

Similarly, 
$\lim _{T \rightarrow \infty}\left\{\left\|\frac{\partial(F u)}{\partial x_{i}}\right\|_{L^{1}\left(\Omega \backslash B_{T}\right)}: i=1, \ldots, n\right\} \leq \lambda d(U)$.

These relations imply that

$d(F U) \leq \lambda d(U)$.

Finally, from (15) and (16) we conclude that $\omega_{0}(F U) \leq \lambda \omega_{0}(U)$.

According to Theorem 1.2, we obtain that the operator $F$ has a fixed point $x$ in $\bar{B}_{r_{0}}$, and thus functional integraldifferential equation (10) has at least one solution in the space $W^{1,1}(\Omega)$.

Now, we present two examples which verify the effectiveness and applicability of Theorem 3.3.

Example 3.4 Consider the following functional integraldifferential equation

$$
\begin{aligned}
u\left(x_{1}, x_{2}, x_{3}\right) & =\sqrt[4]{x_{1}^{5}}+e^{-\left(x_{1}+x_{2}+x_{3}+1\right)} u\left(x_{1}, x_{2}, x_{3}\right) \\
& +\int_{0}^{1} \int_{0}^{1} \int_{0}^{1} \frac{e^{-\left(x_{1}+x_{2}+x_{3}\right)}}{\left(y_{1}+1\right)^{3}\left(y_{2}+2\right)^{2}\left(y_{3}+5\right)} \\
& \times \cos \left(y_{1} u\left(x_{1}, x_{2}, x_{3}\right) \frac{\partial u}{\partial x_{1}}\left(y_{1}, y_{2}, y_{3}\right)+y_{2} \frac{\partial u}{\partial x_{2}}\left(y_{1}, y_{2}, y_{3}\right)\right. \\
& \left.+y_{3} \frac{\partial u}{\partial x_{3}}\left(y_{1}, y_{2}, y_{3}\right)+\frac{1}{2} u\left(y_{1}, y_{2}, y_{3}\right)\right) \mathrm{d} y_{1} \mathrm{~d} y_{2} \mathrm{~d} y_{3} .
\end{aligned}
$$

Eq. (17) is a special case of Eq. (10) with

$$
\begin{aligned}
& \Omega=[0,1] \times[0,1] \times[0,1], p\left(x_{1}, x_{2}, x_{3}\right)=\sqrt[4]{x_{1}^{5}}, \\
& q\left(x_{1}, x_{2}, x_{3}\right)=e^{-\left(x_{1}+x_{2}+x_{3}+1\right)}, \quad g\left(y_{1}, y_{2}, y_{3}, u_{0}, u_{1}, u_{2}, u_{3}, u_{4}\right) \\
& =\cos \left(y_{1} u_{0} u_{1}+y_{2} u_{2}+y_{3} u_{3}+u_{4}\right), T u=\frac{1}{2} u, \\
& k\left(x_{1}, x_{2}, x_{3}, y_{1}, y_{2}, y_{3}\right)=\frac{e^{-\left(x_{2}+y_{2}+y_{3}+1\right)}}{\left(y_{1}+1\right)^{3}\left(y_{2}+2\right)^{2}\left(y_{3}+5\right)}, \\
& g_{1}\left(x_{1}, x_{2}, x_{3}\right)=g_{3}\left(x_{1}, x_{2}, x_{3}\right)=e^{-\left(x_{1}+x_{2}+x_{3}+1\right)},
\end{aligned}
$$

and

$$
g_{2}\left(x_{1}, x_{2}, x_{3}\right)=\frac{1}{\left(y_{1}+1\right)^{2}\left(y_{2}+2\right)\left(y_{3}+5\right)} .
$$

It is easy to see that $p \in W^{1,1}(\Omega), q \in B C^{1}(\Omega)$ and $\lambda=2 e^{-1}$. Also, $g$ satisfies Carathéodory conditions and if we define $a\left(x_{1}, x_{2}, x_{3}\right)=\zeta(x)=1$, then condition (ii) of Theorem 3.3 holds. We observe that $g_{1}, g_{3} \in L^{1}(\Omega), g_{2} \in$ $L^{\infty}(\Omega)$ and $k$ satisfies condition (iii). Moreover, it can be easily shown that each number $r \geq 4$ satisfies the inequality in condition (iv), i.e.,

$$
\begin{aligned}
& \|p\|_{1,1}+\lambda r+M\left\|g_{1}\right\|_{L^{1}(\Omega)}\left\|g_{2}\right\|_{L^{\infty}} \zeta(r) \leq 1+2 e^{-1} r \\
& +\frac{\left(1-e^{-1}\right)^{3}}{10} \leq r .
\end{aligned}
$$

Thus, as the number $r_{0}$ we can take $r_{0}=4$. Consequently, all the conditions of Theorem 3.3 are satisfied. Hence the functional integral-differential equation (17) has at least one solution in the space $W^{1,1}(\Omega)$.

Example 3.5 Consider the following functional integraldifferential equation

$$
u(x)=\frac{u(x)}{x+2}+\int_{0}^{1} \frac{\sqrt[5]{y^{3} u(y)+3 u^{(2)}(y)+u^{(3)}(y)}}{1+\left(u^{\prime}\right)^{2}(y) e^{\sin \left(u^{(2)}(y)+1\right)}} \mathrm{d} y .
$$

Eq. (18) is a special case of Eq. (10) with

$$
p(x)=0, q(x)=\frac{1}{x+2}, k(x, y)=e^{x-y}, T(u)=0, \Omega=[0,1]
$$

and

$$
g\left(y, u_{0}, u_{1}, u_{2}, u_{3}, u_{4}\right)=\frac{\sqrt[5]{y^{3} u_{0}+3 u_{2}+u_{4}}}{1+u_{1}^{2} e^{\sin \left(u_{2}+1\right)}} \cdot T(u)=0
$$

It is easy to see that $q \in B C^{1}(\Omega)$ and $\lambda=\frac{3}{4}$. Also, $g$ satisfies Carathéodory conditions and if we define $a(x)=\sqrt[5]{5}$ and $\zeta(x)=\sqrt[5]{x}$, then condition (ii) of Theorem 3.3 holds. Moreover, $k$ is continuous and has a continuous derivative of order 1 with respect to the first argument. On the other hand, $g_{1}(x)=g_{3}(x)=e^{x}$ and $g_{2}(x)=e^{-x}$. It can be easily shown that each number $r \geq 10$ satisfies the inequality in condition (iv), i.e.,

$$
\begin{aligned}
& \|p\|_{1,1}+\lambda r+M\left\|g_{1}\right\|_{L^{1}(\Omega)}\left\|g_{2}\right\|_{L^{\infty}} \zeta(r) \leq \frac{3}{4} r+\sqrt[5]{5}(e-1) \\
& \left(1-e^{-1}\right) \sqrt[5]{r} \leq r
\end{aligned}
$$

Hence, as the number $r_{0}$ we can take $r_{0}=10$. Consequently, all the conditions of Theorem 3.3 are satisfied. It implies that the functional integral-differential equation (18) has at least one solution in the space $W^{1,1}(\Omega)$.

Open Access This article is distributed under the terms of the Creative Commons Attribution 4.0 International License (http://creative commons.org/licenses/by/4.0/), which permits unrestricted use, distribution, and reproduction in any medium, provided you give appropriate credit to the original author(s) and the source, provide a link to the Creative Commons license, and indicate if changes were made.

\section{References}

1. Agarwal, R.P., Benchohra, M., Seba, D.: On the application of measure of noncompactness to the existence of solutions for fractional differential equations. Results Math. 55, 221-230 (2009)

2. Aghajani, A., Allahyari, A., Mursaleen, M.: A generalization of Darbo's theorem with application to the solvability of systems of integral equations. J. Comput. Appl. Math. 260, 68-77 (2014) 
3. Aghajani, A., Banaś, J., Jalilian, Y.: Existence of solutions for a class of nonlinear Volterra singular integral equations. Comput. Math. Appl. 62, 1215-1227 (2011)

4. Aghajani, A., O'Regan, D., Shole Haghighi, A.: Measure of noncompactness on $L^{p}\left(\mathbb{R}^{n}\right)$ and applications. Cubo A Math. J. 17, 85-97 (2015)

5. Arab, R., Allahyari, R., Shole Haghighi, A.: Construction of measures of noncompactness of $C^{k}(\Omega)$ and $C_{0}^{k}$ and their application to functional integral-differential equations. Bull. Iran. Math. Soc. 43(1), 53-67 (2017)

6. Ayad, A.: Spline approximation for first order Fredholm delay integro-differential equations. Int. J. Comput. Math. 70, 467-476 (1999)

7. Banaś, J.: Measures of noncompactness in the study of solutions of nonlinear differential and integral equations. Cent. Eur. J. Math. 10(6), 2003-2011 (2012)

8. Banaś, J., Goebel, K.: Measure of noncompactness in Banach spaces. Lecture notes in pure and applied mathematics, vol. 60. Dekker, New York (1980)

9. Banaś, J., O'Regan, D., Sadarangani, K.: On solutions of a quadratic hammerstein integral equation on an unbounded interval. Dynam. Syst. Appl. 18, 251-264 (2009)

10. Behiry, S.H., Hashish, H.: Wavelet methods for the numerical solution of Fredholm integro-differential equations. Int. J. Appl. Math. 11, 27-35 (2002)

11. Brezis, H.: Functional analysis. Sobolev spaces and partial differential equations. Springer Science, Business Media, LLC, New York (2011)

12. Bloom, F.: Asymptotic bounds for solutions to a system of damped integro-differential equations of electromagnetic theory. J. Math. Anal. Appl. 73, 524-542 (1980)

13. Darbo, G.: Punti uniti in trasformazioni a codominio non compatto. Rend. Sem. Mat. Univ. Padova. 24, 84-92 (1955)

14. Drabek, P., Milota, J.: Methods of nonlinear analysis. Birkhauser Velgar AG, Basel (2007)

15. Darwish, M.A.: On monotonic solutions of a quadratic integral equation with supremum. Dynam. Syst. Appl. 17, 539-550 (2008)

16. Darwish, M.A., Henderson, J., O'Regan, D.: Existence and asymptotic stability of solutions of a perturbed fractional functional-integral equation with linear modification of the argument. Bull. Korean Math. Soc. 48, 539-553 (2011)

17. Dhage, B.C., Bellale, S.S.: Local asymptotic stability for nonlinear quadratic functional integral equations. Electron. J. Qual. Theory Differ. Equ. 10, 1-13 (2008)
18. Forbes, L.K., Crozier, S., Doddrell, D.M.: Calculating current densities and fields produced by shielded magnetic resonance imaging probes. SIAM J. Appl. Math. 57, 401-425 (1997)

19. Garey, L.A., Gladwin, C.J.: Direct numerical methods for first order Fredholm integro-differential equations. Int. J. Comput. Math. 34, 237-246 (1990)

20. Hanche-Olsen, H., Holden, H.: The Kolmogorov-Riesz compactness theorem. Expo. Math. 28, 385-394 (2010)

21. Holmaker, K.: Global asymptotic stability for a stationary solution of a system of integro-differential equations describing the formation of liver zones. SIAM J. Math. Anal. 24, 116-128 (1993)

22. Hosseini, S.M., Shahmorad, S.: Tau numerical solution of Fredholm integro-differential equations with arbitrary polynomial base. Appl. Math. Model. 27, 145-154 (2003)

23. Jleli, M., Mursaleen, M., Sadarangani, K., Samet, B.: A cone measure of noncompactness and some generalizations of Darbo's theorem with applications to functional integral equations. J. Funct. Spaces 9896502, 11 (2016)

24. Kanwal, R.P.: Linear integral differential equations theory and technique. Academic Press, New York (1971)

25. Kuratowski, K.: Sur les espaces complets. Fund. Math. 15, 301-309 (1930)

26. Liu, L., Guo, F., Wu, C., Wu, Y.: Existence theorems of global solutions for nonlinear Volterra type integral equations in Banach spaces. J. Math. Anal. Appl. 309, 638-649 (2005)

27. Mursaleen, M., Rizvi, S.M.H.: Solvability of infinite system of second order differential equations in $c_{0}$ and $l_{1}$ by Meir-Keeler condensing operator. Proc. Am. Math. Soc. 144(10), 4279-4289 (2016)

28. Mursaleen, M., Mohiuddine, S.A.: Applications of noncompactness to the infinite system of differential equations in $l_{p}$ spaces. Nonlinear Anal. 75(4), 2111-2115 (2012)

29. Olszowy, L.: Solvability of infinite systems of singular integral equations in Fréchet space of coninuous functions. Comp. Math. Appl. 59, 2794-2801 (2010)

\section{Publisher's Note}

Springer Nature remains neutral with regard to jurisdictional claims in published maps and institutional affiliations. 